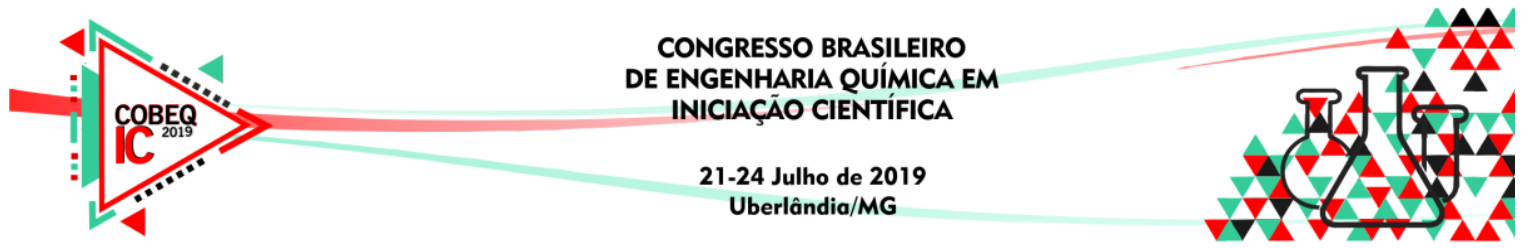

\title{
LIXIVIAÇÃO BACTERIANA DE METAIS: UMA ABORDAGEM DA MODELAGEM POR ESPAÇO DE ESTADOS DE UM REATOR PARA O PROCESSO CONTÍNUO
}

\author{
F. P. SILVA ${ }^{1}$, T. C. O. SAMPAIO ${ }^{1}$, A. G. CRUZ ${ }^{1}$, R. M. AGUIAR ${ }^{1}$ e M. G. F. MORAES ${ }^{1}$ \\ ${ }^{1}$ Universidade Federal do Rio de Janeiro, Escola de Química \\ E-mail para contato: silva_fp@eq.ufrj.br
}

\begin{abstract}
RESUMO - A mineração representa, atualmente, cerca de 4\% do Produto Interno Bruto (PIB) nacional. Diversos produtos são obtidos a partir destas atividades e abastecem as indústrias de geração de energia, de bens de consumo, metalúrgica, entre outras. Além disso, metais como cobre, cobalto, ouro e urânio possuem alto valor agregado, o que coloca este setor num patamar de extrema importância. Embora seja assim, a exploração de minérios de baixos teores ainda requer investimento em novas tecnologias, como é o caso da biolixiviação, processo onde um microrganismo auxilia na extração de metais minérios ou resíduos ricos em metal, levando sulfetos a sulfatos e produzindo ácido sulfúrico que solubiliza, inclusive, o metal disponível. Devido à necessidade da compreensão da aplicação da modelagem dinâmica de processos na indústria mineradora, o presente trabalho modela um reator CSTR para operar continuamente um processo de lixiviação bacteriana, avaliando perturbações do tipo degrau unitário na Concentração de substrato $(\mathrm{S})$, células $(\mathrm{X})$ e produto $(\mathrm{P})$. Através de uma rotina no software MATLAB®, as funções de transferência do processo foram obtidas e utilizadas para verificar a influência de perturbações degrau unitário quando aplicadas às variáveis de entrada na saída do processo.
\end{abstract}

\section{INTRODUÇÃO}

A mineração pode ser definida como a extração, elaboração e beneficiamento de minerais. Atualmente, com uma exploração que resulta numa produção que compõe algo em torno de $4 \%$ do Produto Interno Bruto (PIB) brasileiro e produtos que abastecem as indústrias de geração de energia, bens de consumo, metalúrgica, entre outras, a indústria extrativa mineral é um dos setores estratégicos mais relevantes (SANTOS, 2010; DNPM, 2015).

De acordo com Silva (2014), uma das grandes preocupações tem sido a exploração de minérios de baixos teores. Neste cenário, surge a lixiviação bacteriana, ou biolixiviação, técnica onde utilizam-se microrganismos na extração a partir destes minérios e/ou resíduos oriundos de mineração (WATLING, 2008; SOBRAL et al., 2011). No processo, oxidam-se sulfetos a sulfatos e produz-se ácido sulfúrico como consequência. Apresentando como vantagens a menor demanda de energia, economia de insumos utilizados, diminuição na geração de efluentes e maior eficiência de extração de metais deste tipo de minério (GERICKE et al., 


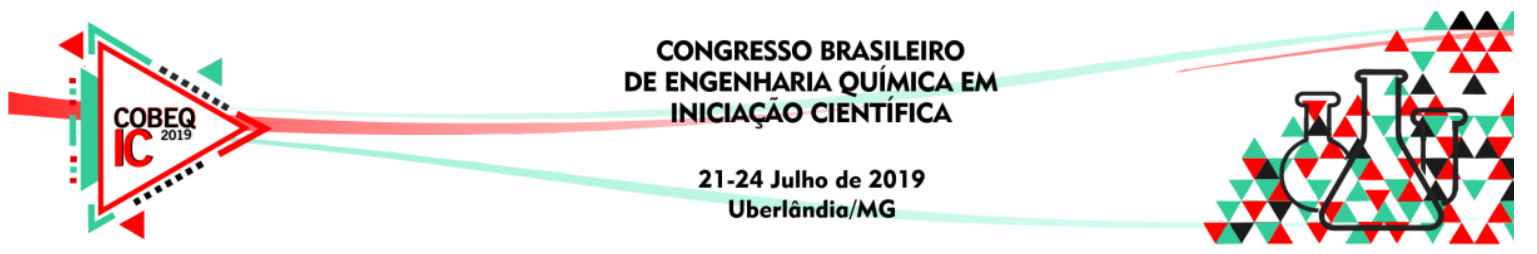

2011), a biolixiviação pode acontecer em depósitos ou tanques agitados (KARAVAIKO et al., 1988).

Conforme Schippers (2007), os microrganismos que realizam o processo podem ser classificados de acordo com a temperatura em que se desenvolvem como mesófilos (até $40^{\circ} \mathrm{C}$ ) e termófilos moderados $\left(40-55^{\circ} \mathrm{C}\right)$ e extremos $\left(55-80^{\circ} \mathrm{C}\right)$, sendo as espécies Acidithiobacillus ferrooxidans, Acidithiobacillus thiooxidans e Leptospirillum ferrooxidans, as bactérias mesófilas mais frequentemente isoladas em ambientes de lixiviação. Falando-se especificamente da Acidithiobacillus ferrooxidans, é sabido que ela tem resistência natural a uma gama de íons metálicos e cresce à temperatura ambiente, obtendo energia a partir da oxidação de compostos de enxofre e íons ferrosos a íons férricos (DONATI et al., 2007), trabalhando bem na faixa de pHs entre 1,0-4,0 (WATLING, 2008).

Na literatura, a modelagem dinâmica desses processos, para fins de análise e controle não é tão estudada. Utilizando parâmetros cinéticos e valores de regime de processo descritos por Pesic (1989) e Tavares (2015), a avaliação da dinâmica desse processo foi obtida. Assim, o objetivo do presente trabalho se dá pela modelagem por espaço de estados e avaliação da dinâmica da biolixiviação em reator CSTR da pirita utilizando a A. ferrooxidans.

\section{METODOLOGIA}

\subsection{O processo}

Foi utilizado como referência o processo descrito por d'Hugues et al. (2008). Considerouse que o mesmo ocorre dentro de um reator CSTR (Figura 1). A concentração de células de $A$. ferrooxidans é inicialmente $3,33 \mathrm{~g}$ células $\cdot \mathrm{L}^{-1}$. No reator, há entrada contínua de pirita $\left(\mathrm{FeS}_{2}\right)$ na granulometria de 53-63 $\mu \mathrm{m}$ suspensa no meio de cultivo, formando-se uma polpa. Volume (V) mantido constante em 15,0 L a uma vazão de entrada $(F)$ da polpa de inicialmente 2,78 10${ }^{4} \mathrm{~L}^{-1}{ }^{-1}$, também constante. A concentração de minério na corrente de alimentação $\left(S_{0}\right)$ possui valor inical de $133,33 \mathrm{~g} \cdot \mathrm{L}^{-1}$. Processo à temperatura ambiente e constante, com $\mathrm{pH}$ controlado entre 1,5-1,8. Concentração do substrato $\left(\mathrm{FeS}_{2}\right)$ é indicada por $S$. Todo substrato consumido vai a produto porém, parte não reagida sai na corrente de saída. Sulfato de ferro (II) $\left(\mathrm{FeSO}_{4}\right)$ é o produto de interesse, sendo $P$ a sua concentração.

Figura 1 - Processo de lixiviação bacteriana ou, simplesmente, biolixiviação.

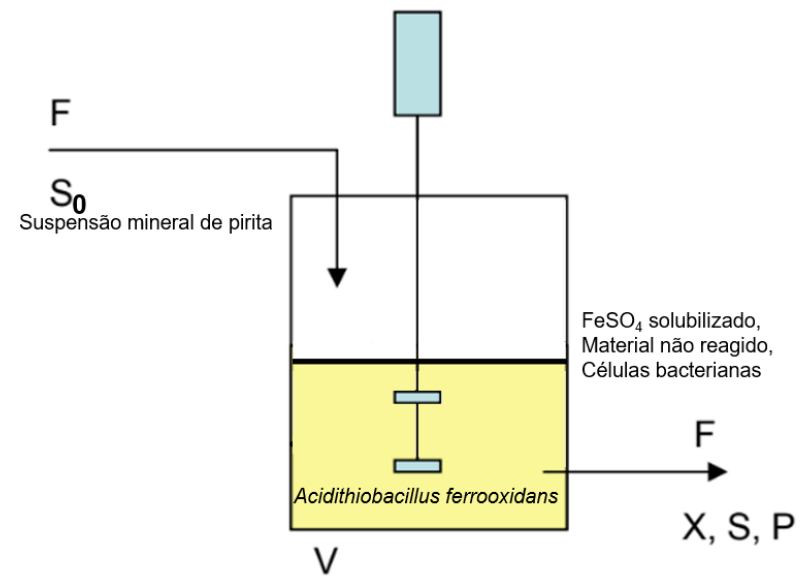




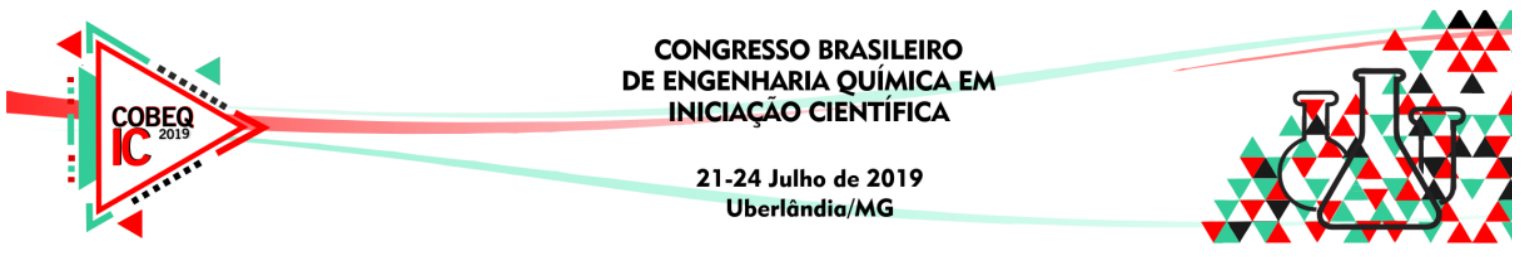

\subsection{Balanços materiais para o reator}

Para os balanços de massa por componente (BMCs) do bioprocesso, faz-se necessário utilizar um balanço de massa para as células (BMX), um para o substrato $\left(\mathrm{FeS}_{2}\right)(\mathrm{BMS})$ e outro para o produto principal $\left(\mathrm{FeSO}_{4}\right)(\mathrm{BMP})$, a fim de simplificação dos cálculos.

Considerando $\mathrm{V}$ constante, e a taxa de crescimento de células $r_{X}=\mu X$, sendo o termo $\mu$ a taxa de crescimento específico da bactéria provindo da equação de Monod (BORZANI et al., 2001) e a taxa de consumo de substrato $-r_{S}=k X S_{0} p_{O_{2}} \exp \left(-\frac{E_{a}}{R T}\right)$ (PESIC et al., 1989), as Equações (1), (2) e (3) mostram os BMX, BMS e BMP, respectivamente.

$$
\begin{aligned}
& \frac{d(X V)}{d t}=-(F X)+r_{X} V \rightarrow \frac{d X}{d t}=\frac{\mu_{\text {máx }} S}{\left(k_{S}+S\right)} X-\frac{F}{V} X \\
& \frac{d(S V)}{d t}=\left(S_{0}-S\right) * F-\left(-r_{S}\right) \rightarrow \frac{d S}{d t}=\left(S_{0}-S\right) \frac{F}{V}-\left[k X S_{0} p_{O_{2}} \exp \left(-\frac{E_{a}}{R T}\right)\right] \\
& \frac{d(P V)}{d t}=-F P+r_{P} V \rightarrow \frac{d P}{d t}=\left[k X S_{0} p_{O_{2}} \exp \left(-\frac{E_{a}}{R T}\right)\right]-\frac{F}{V} P
\end{aligned}
$$

$\mathrm{Na}$ determinação dos parâmetros nas condições do processo e outras constantes envolvidas nas equações, foi utilizado dados disponíveis na literatura, contidos na Tabela 1.

Tabela 1 - Parâmetros e constantes envolvidas nas equações de taxa

\begin{tabular}{|c|c|c|c|c|}
\hline $\begin{array}{c}\text { Parâmetros } \\
\text { do processo }\end{array}$ & Nome & Valor & Unidade & Referência \\
\hline$S_{0}$ & Concentração de substrato inicial & 133,3 & $\mathrm{~g} . \mathrm{L}^{-1}$ & Pesic et al. (1989) \\
\hline$k$ & Velocidade específica de reação & $4,50 \times 10^{7}$ & $\mathrm{~L}^{-1}{ }^{-1} \cdot \mathrm{s}^{-1}$ & Pesic et al. (1989) \\
\hline$p_{O 2}$ & Pressão parcial do oxigênio & 0,21 & - & Pesic et al. (1989) \\
\hline$E_{a}$ & Energia de Ativação & $-58,77 \times 10^{3}$ & $\mathrm{~J} . \mathrm{mol}^{-1}$ & Pesic et al. (1989) \\
\hline$T$ & Temperatura & 298,15 & $\mathrm{~K}$ & Pesic et al. (1989) \\
\hline$\mu_{\text {máx }}$ & Taxa máxima de crescimento bacteriano & $2,50 \times 10^{-5}$ & $\mathrm{~s}^{-1}$ & Tavares et al. (2015) \\
\hline$k_{S}$ & Constante de saturação & 2 & $\mathrm{~g} . \mathrm{L}^{-1}$ & Tavares et al. (2015) \\
\hline
\end{tabular}

\subsection{Modelagem do reator por espaço de estados}

Para a modelagem por espaço de estados, fez-se a linearização dos termos não lineares dos BMCs, sendo as variáveis de estado do processo $X, S$ e $P$ e as variáveis de entrada $S_{0}$ e $F$. As Equações (4) e (5) são representações do modelo dinâmico na forma matricial via espaço de estados sendo (4) a equação do estado e (5) a equação da saída.

$$
\underline{\dot{x}}=\underline{f}(\underline{x}, \underline{u}) \approx(\overline{\bar{A}} \underline{x}+\overline{\bar{B}} \underline{u}))
$$




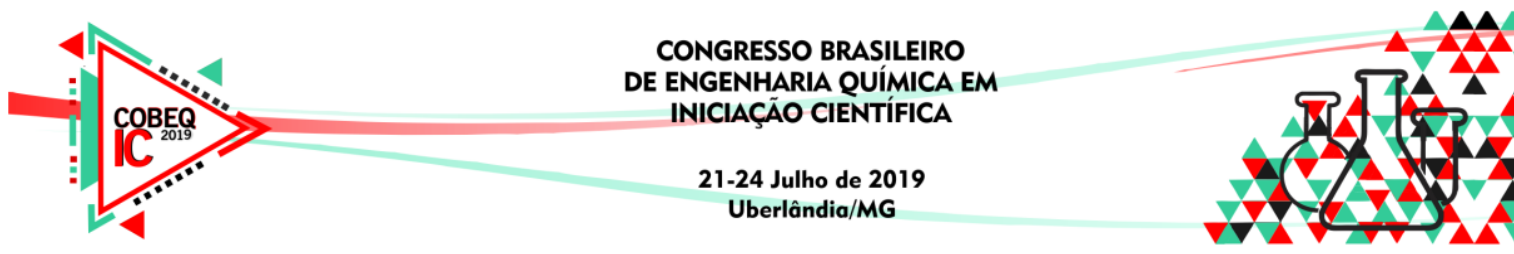

$$
\underline{y}=\underline{f}(\underline{x}, \underline{u})=(\overline{\bar{C}} \underline{x}+\overline{\bar{D}} \underline{u})
$$

As matrizes $\overline{\bar{A}}$ e $\overline{\bar{B}}$ são matrizes jacobianas, formadas pelas derivadas parciais dos BMCs em relação às variáveis de estado e às variáveis de entrada, respectivamente, avaliadas no estado estacionário $(S S)$ inicial. O SS inicial foi determinado resolvendo-se o sistema algébrico resultante das equações (1) a (3) quando os termos de acúmulo são nulos. Adotando-se BMX, BMS e BMP por, respectivamente $f_{1}, f_{2}$ e $f_{3}, \overline{\bar{A}}$ e $\overline{\bar{B}}$ são definidas pelas Equações (6) e (7). As variáveis de saída foram definidas como iguais aos estados $(\underline{y}=\underline{x}) \mathrm{e}$, portanto, $\overline{\bar{C}}$ é a matriz identidade e $\overline{\bar{D}}$ é matriz nula.

$$
\begin{aligned}
& \overline{\bar{A}}=\left[\begin{array}{lll}
\left(\frac{\partial f_{1}}{\partial X}\right)_{S S} & \left(\frac{\partial f_{1}}{\partial S}\right)_{S S} & \left(\frac{\partial f_{1}}{\partial P}\right)_{S S} \\
\left(\frac{\partial f_{2}}{\partial X}\right)_{S S} & \left(\frac{\partial f_{2}}{\partial S}\right)_{S S} & \left(\frac{\partial f_{2}}{\partial P}\right)_{S S} \\
\left(\frac{\partial f_{3}}{\partial X}\right)_{S S} & \left(\frac{\partial f_{3}}{\partial S}\right)_{S S} & \left(\frac{\partial f_{3}}{\partial P}\right)_{S S}
\end{array}\right]=\left[\begin{array}{ccc}
\mu-\frac{F}{V} & \frac{\mu_{\text {máx }} \cdot k_{S}}{\left(k_{S}+S\right)^{2}} & 0 \\
-\left[k X S_{0} p_{O_{2}} \exp \left(-\frac{E_{a}}{R T}\right)\right. & -\frac{F}{V} & 0 \\
{\left[k X S_{0} p_{O_{2}} \exp \left(-\frac{E_{a}}{R T}\right)\right.} & 0 & -\frac{F}{V}
\end{array}\right]_{S S} \\
& \overline{\bar{B}}=\left[\begin{array}{ll}
\left(\frac{\partial f_{1}}{\partial S_{0}}\right)_{S S} & \left(\frac{\partial f_{1}}{\partial F}\right)_{S S} \\
\left(\frac{\partial f_{2}}{\partial S_{0}}\right)_{S S} & \left(\frac{\partial f_{2}}{\partial F}\right)_{S S} \\
\left(\frac{\partial f_{3}}{\partial X}\right)_{S S} & \left(\frac{\partial f_{3}}{\partial F}\right)_{S S}
\end{array}\right]=\left[\begin{array}{cc}
0 & -\frac{X}{V} \\
\frac{F}{V} & \frac{S 0-S}{V} \\
0 & -\frac{P}{V}
\end{array}\right]_{S S}
\end{aligned}
$$

Com as matrizes definidas, foram obtidas a matriz $\overline{\bar{G}}(s)$ de funções de transferência (FT), utilizando o software MATLAB ${ }^{\circledR}$ e estudou-se dinâmica frente a perturbações do tipo degrau em $S_{0}$ e $F$ quando aplicadas às saídas do processo.

\section{RESULTADOS E DISCUSSÃO}

Com o auxílio da rotina implementada no MATLAB ${ }^{\circledR}$, as FT foram obtidas. A partir da transformação do espaço de estados para FT, foram encontradas as FT que refletem à dinâmica entre $X, S$ e $P$ com relação a $S_{0}$ e em $F$, em variáveis desvio. A perturbação degrau adotada para as variáveis de entrada foi dada no instante $\mathrm{t}=0$. Na Figura 2 constam-se as respostas dinâmicas, bem como as FT obtidas para cada caso. As FT estão sendo identificadas de $G 1(s)$ a $G 6(s)$, onde $G 1(s), G 3(s)$ e $G 5(s)$ correspondem ao degrau unitário em $S_{0}$ e foram multiplicadas pelo fator $10^{2}$ para se enquadrarem em cada gráfico, $G 2(s), G 4(s)$ e $G 6(s)$ dizem respeito à mesma perturbação em $F$.

É válido ressaltar que as FT encontradas na forma de polos-zeros, possuem equação característica $(s+0,667)\left(s^{2}+0,06667 s+0,02573\right)$, possuindo um polo real negativo e dois polos complexos de parte real negativa, conferindo às respostas um caráter oscilatório que chegará a um novo patamar estável.

Analisando-se os resultados para $X$, primeiramente para a dinâmica em relação a $S_{0}$, observa-se a presença de um zero negativo igual a um dos polos, de modo a se ter um cancelamento polo-zero, e o processo resultante se dá puramente por de segunda ordem 


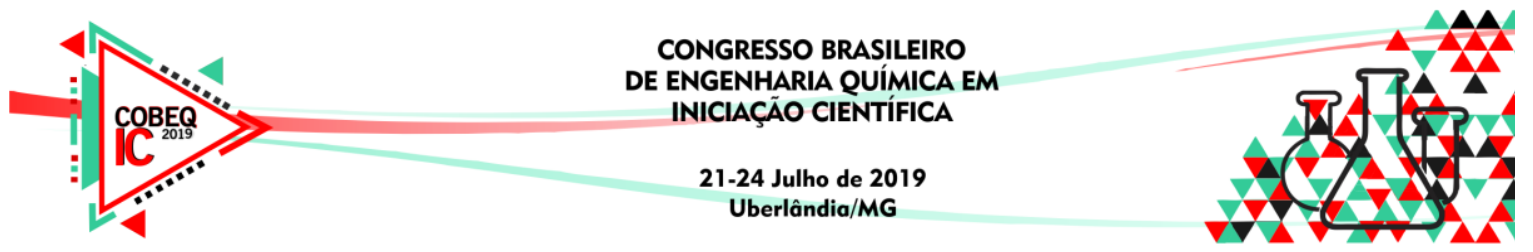

subamortecido. Em relação a $F$, a FT possui um zero positivo, indicando a existência de resposta inversa, observada pelo antagonismo entre dois fenômenos: um primeiro, de dinâmica mais rápida, associado ao fato de que, com aumento de $F$, há o efeito de diluição; outro, de dinâmica principal, fazendo com que $X$ voltasse a aumentar pela maior disponibilidade de $S$.

Figura 2 - Respostas de $X, S$ e $P$ ao degrau unitário, bem como as FT correspondentes.
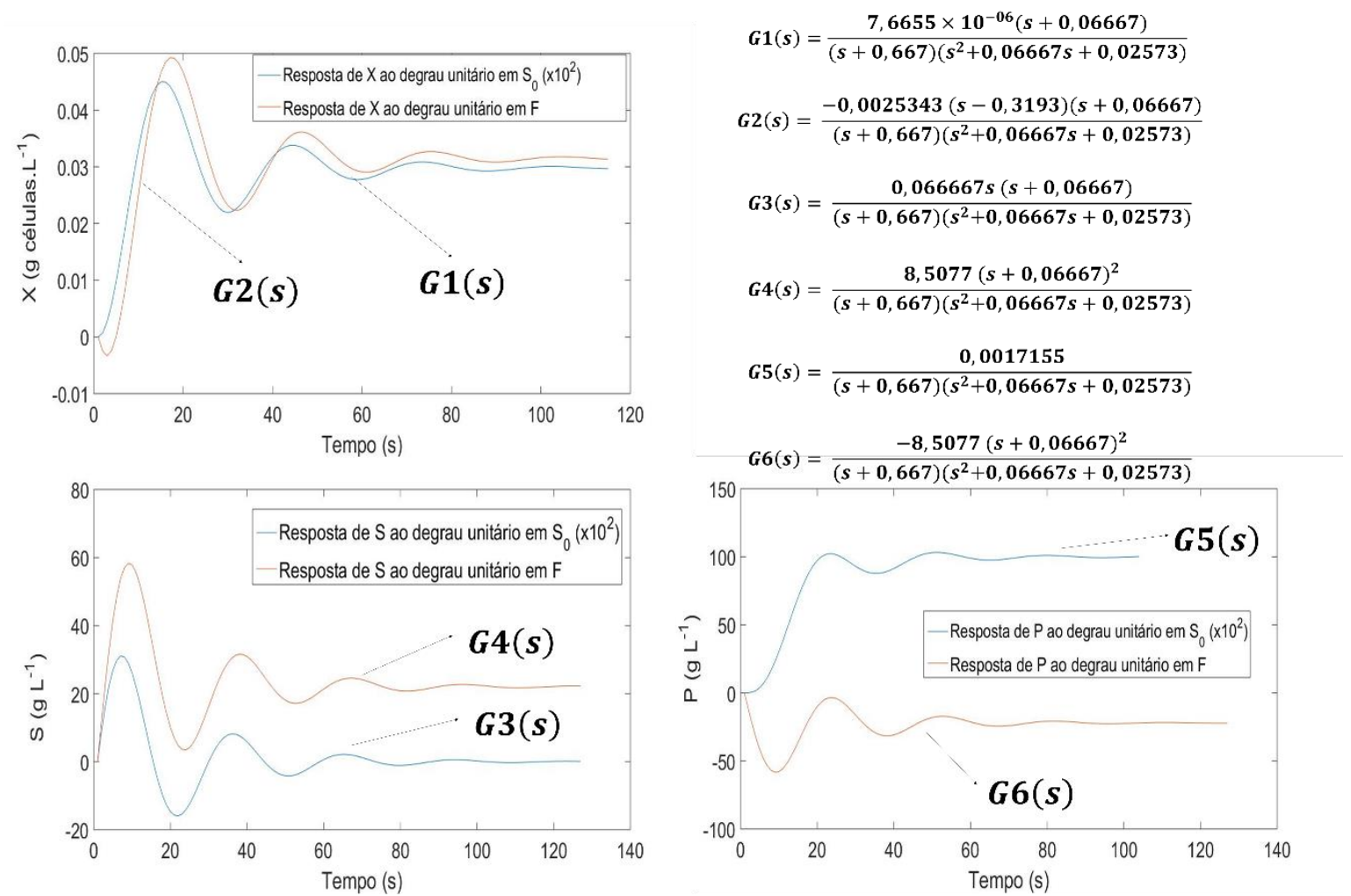

No caso de $S$, para um degrau unitário em $F$, percebe-se um overshoot mais pronunciado do que o overshoot intrínseco de um processo de segunda ordem, visto a presença de dois zeros negativos repetidos (mesmo sendo um cancelado a um dos polos). Um degrau em $S_{0}$ mostra comportamento semelhante ao anterior. Para a dinâmica de $P$, com relação a $S_{0}$, não se observa dinâmica no numerador, possuindo um caráter menos oscilatório (menos potencializado o efeito de overshoot) que os outros casos, podendo essa FT ser associada puramente a um processo de $1^{\mathrm{a}}$ ordem em série a um de $2^{\mathrm{a}}$ ordem subamortecido. Em relação a $F$, há existência de ganho negativo para $P$, indicando que um aumento da vazão, faz com que a produtividade diminua, visto menor tempo de residência dentro do reator; para esse caso, pela existência de zero negativo, o efeito de overshoot é também mais pronunciado.

\section{CONCLUSÃO}

No presente trabalho, foi possível determinar um modelo que descrevesse a dinâmica de um reator CSTR para um processo contínuo de biolixiviação, linearizá-lo e representá-lo na 


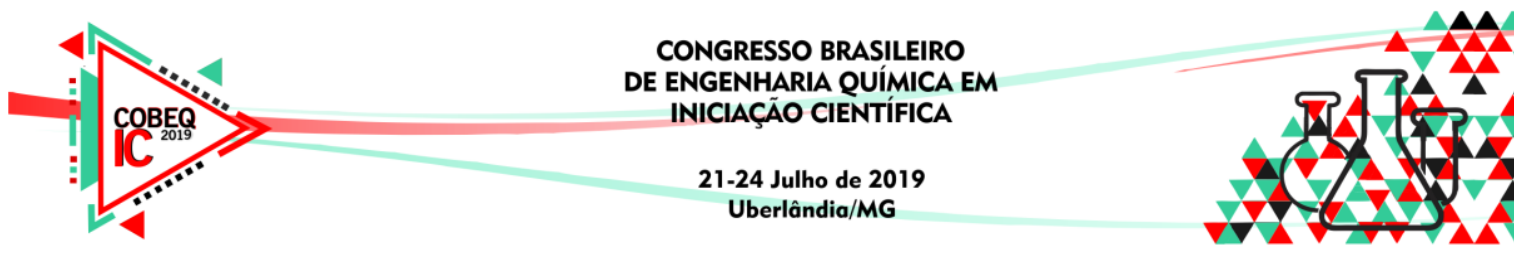

forma de espaço de estado, de modo a avaliar a dinâmica desse processo utilizando a abordagem por funções de transferência. A devida descrição da dinâmica das respostas de $X, S$ e $P$ a um degrau unitário em $S_{0}$ e $F$ foi obtida. Como trabalhos futuros, sugere-se a comparação dos resultados obtidos a resultados para curvas de reação da planta (processo real), bem como investigação de possíveis estratégias de controle para esse processo.

\section{REFERÊNCIAS}

BORZANI, Q.; LIMA, U.A.; AQUARONE, E. Biotecnologia Industrial, v. 2, Editora Edgar Blucher, 2001.

D'HUGUES, P.; JOULIAN, C.; SPOLAORE, P.; MICHEL, C.; GARRIDO, F.; MORIN, D. Continuous bioleaching of a pyrite concentrate in stirred reactors: Population. Hydrometallurgy, v. 94, p. 34-41, 2008.

DNPM (Brasília) - Departamento Nacional de Produção Mineral. Sumário 2015, v. 34, 2015.

DONATI, E. R.; SAND, W. Microbial processing of metal sulfides. La Plata: Springer, Cap. 1, p. 3-33, 2007.

GERICKE, M.; GOVENDER, Y. Bioleaching strategies for the treatment of nickel-copper sulphide concentrates. Minerals Engineering, v. 24, p. 1106-1112, 2011.

KARAVAIKO, G. I. Biogeotechnology of Metals-Manual. Center for Internal Projects, MOSCOW, 1988.

PESIC, B.; OLIVER, D.J.; WICHLACZ, P. An Electrochemical Method of Measuring the Oxidation Rate of Ferrous to Ferric Iron with Oxygen in the Presence of Thiobacillus ferrooxidans. Biotechnology and Bioengineering, v. 33, p. 428-439, 1989.

SANTOS, L. G. S.; OLIVEIRA, D. M. Biolixiviação: Utilização de Micro-organismos na Extração de Metais. Série Tecnologia Ambiental, CETEM/MCTI, 2010.

SCHIPPERS, A.; SAND, W. Bacterial Leaching of Metal Sulfides Proceeds by Two Indirect Mechanisms via Thiosulfate or via Polysulfides and Sulfur. Applied And Environmental Microbiology, v. 65, p. 319-321, 1999.

SILVA, M. J. S. Materiais \& Mineração. Aliança do Conhecimento. Revista ABM Metalurgia, v. 70, 2014.

SOBRAL, L. G. S.; OLIVEIRA, D. M.; SOUZA, C. E. G. Biohydrometallurgical Processes: A practical Approach. CETEM/MCTI, 2011.

TAVARES, H.; FELTRIN, A.; ANGIOLETTO, E.; MENDES, E.; MENDES, E. Análise de engenharia metabólica para o desenvolvimento e maximização quantitativa do tratamento de drenagem ácida de mineração para produção de $\mathrm{Fe}^{3+}$ e ácido sulfúrico, gerados pelo metabolismo da bactéria Acidithiobacillus ferrooxidans. Ciência e Tecnologia, 2015.

WATLING, H.R. The bioleaching of nickel-copper sulfides. Hydrometallurgy, v. 91, p. 70-88, 2008. 